\title{
On Markov Moment Problem and Mazur-Orlicz Theorem
}

\author{
Octav Olteanu' ${ }^{1}$ Janina Mihaela Mihăilă \\ ${ }^{1}$ Department of Mathematics-Informatics, Faculty of Applied Sciences, Politehnica University of Bucharest, Bucharest, Romania \\ ${ }^{2}$ Department of Engineering Sciences, Ecological University of Bucharest, Bucharest, Romania \\ Email: olteanuoctav@yahoo.ie, janinamihaelamihaila@yahoo.it
}

How to cite this paper: Olteanu, O. and Mihăilă, J.M. (2017) On Markov Moment Problem and Mazur-Orlicz Theorem. Open Access Library Journal, 4: e3950. https://doi.org/10.4236/oalib.1103950

Received: October 19, 2017

Accepted: October 22, 2017

Published: October 25, 2017

Copyright (c) 2017 by authors and Open Access Library Inc.

This work is licensed under the Creative Commons Attribution International License (CC BY 4.0).

http://creativecommons.org/licenses/by/4.0/

(c) (†) Open Access

\begin{abstract}
Applications of the generalization of Mazur-Orlicz theorem to concrete spaces are proved. Suitable moment problems are solved, as applications of extension theorems of linear operators with a convex and a concave constraint. In particular, a relationship between Mazur-Orlicz theorem and Markov moment problem is partially illustrated. In the end of this work, an application to the multidimensional Markov moment problem of an earlier extension result on a distanced subspace with respect to a bounded convex set is proved. Contrary to preceding results based on this theorem, now the solution is defined on a space of continuous functions vanishing at the origin. Most of the solutions are operator valued, respectively function valued.
\end{abstract}

\section{Subject Areas}

Functional Analysis, Moment Problem, Mazur-Orlicz Theorem

\section{Keywords}

Markov Moment Problem, Inequalities, Convex Subsets,

Hahn-Banach Principle, Concrete Spaces

\section{Introduction}

Using Hahn-Banach results and Mazur-Orlicz theorem in various applications (the moment problem, flows in infinite networks, transport problems, economic problems) is a useful technique (see [1] [2] and the references therein). In [3] [4] [5] [6] [7], more results on Mazur-Orlicz theorem and the moment problem have been stated or (and) proved. The present work can be regarded as a continuation of the study from the latter works. Most of these results are based on extension theorems for linear operators, with two constraints (one of which is 
convex; the other one is concave). In the first part of this work, applications of a variant of Mazur-Orlicz theorem to concrete spaces are studied. In the second part, we solve appropriate moment problems. In most of the cases, the target-space of the solution is a space of self-adjoint operators or a function space. These are interpolation problems, with two constraints. The lower constraint is sometimes the positivity of the solution. The classical moment problem is an interpolation problem, involving the positivity of the linear functional (or operator) solution. In the case of a Markov moment problem, an "upper domination" condition appears additionally. The latter constraint controls the norm of the solution. In Mazur-Orlicz problems, the interpolation conditions are replaced by inequalities. Both these problems are Hahn-Banach type results (see [8] [9] [10]). The main results of the second part (section 3) of this work are Theorems 3.3, 3.5. The relationship between the Mazur-Orlicz and the corresponding moment problem is illustrated by means of Theorems 2.2, 3.2 of the present work. In time, different connections of the moment problem with several other fields have been pointed out. For example, in [11], one makes the connection to elements of fixed point theory. For the construction of a solution starting from its moments, see [6]. The same paper [6] contains connections between Markov moment problem and extreme points (Krein-Milman theorem). In solving moment problem, three aspects are studied: the existence, the uniqueness and the construction of the solution. Most of the results appearing in the present work refer to the existence of the solution. The interested reader may find results on the uniqueness of the solution in [12] [13]. The background of this work is partially based on some chapters from [14] [15] [16]. The rest of the paper is organized as follows. Section 2 contains two applications of Mazur-Orlicz theorem. In Section 3, some Markov moment problems involving concrete spaces are solved. One of these results (Theorem 3.2) is somehow related to the corresponding similar (last) statement of Section 2 (Theorem 2.2). Another theorem refers to a general extension result involving a vector subspace which is distanced with respect to a convex bounded set. The existence of a multiplicative solution on a space of continuous functions vanishing at the origin is deduced.

\section{Applications of Mazur-Orlicz Theorem}

We start this section by recalling the following variant of the Mazur-Orlicz Theorem [10]. This is a consequence of a Hahn-Banach type result.

Theorem 2.1. (Theorem 5 [10]). Let $X$ be a preordered linear space, $Y$ an order-complete vector lattice, $\left\{x_{j} ; j \in J\right\} \subset X,\left\{y_{j} ; j \in J\right\} \subset Y$ given finite or infinite families of elements. Let $P: X \rightarrow Y$ be a sublinear operator.

The following statements are equivalent

1) there exists a linear operator $F \in L(X, Y)$ such that

$$
F\left(x_{j}\right) \geq y_{j} \forall j \in J, F(x) \geq 0 \forall x \in X_{+}, F(x) \leq P(x) \forall x \in X
$$

2) for any finite subset $J_{0} \subset J$ and any $\left\{\lambda_{j} ; j \in J_{0}\right\} \subset R_{+}$, we have: 


$$
\sum_{j \in J_{0}} \lambda_{j} x_{j} \leq x \Rightarrow \sum_{j \in J_{0}} \lambda_{j} y_{j} \leq P(x)
$$

The next result of this Section uses the order relation given by the coefficients in spaces of analytic functions. On the other hand, let $H$ be a complex Hilbert $U_{0} \in \mathrm{A}(H)$ a selfadjoint operator from $H$ into $H$. One defines

$$
\begin{aligned}
& Y_{1}=\left\{U \in \mathrm{A}(H) ; U U_{0}=U_{0} U\right\}, Y=\left\{U \in Y_{1} ; U V=V U \forall V \in Y_{1}\right\}, \\
& Y_{+}=\{U \in Y ;\langle U(h), h\rangle \geq 0 \forall h \in H\}
\end{aligned}
$$

Obviously, $Y$ defined by (2.3) is a commutative algebra of selfadjoint operators. Moreover, $Y$ is a vector lattice, being complete with respect to the order relation (cf. [14]), and the operatorial norm on $Y$ is solid:

$$
|U| \leq|V| \Rightarrow\|U\| \leq\|V\|, U, V \in Y
$$

The next result is an application of Theorem 2.1 to the space $X$ of all absolutely convergent power series in the disc $|z|<r$, continuous up to the boundary, with real coefficients. The order relation is given by the coefficients: we write

$$
\sum_{n \in \mathrm{N}} \lambda_{n} z^{n} \prec \sum_{n \in \mathrm{N}} \gamma_{n} z^{n} \Leftrightarrow\left(\lambda_{n} \leq \gamma_{n}, \forall n \in \mathrm{N}\right)
$$

Denote $\varphi_{n}(z)=z^{n}, n \in \mathrm{N},|z| \leq r$. Let $Y$ be the space defined by (2.3), $\left(B_{n}\right)_{n \in \mathrm{N}}$ a sequence in $Y$, and $U \in Y$ such that $\|U\|<r$.

\section{Proposition 2.1. Consider the following statements}

1) there exists a linear positive bounded operator $F \in L_{+}(X, Y)$, such that

$$
\begin{gathered}
F\left(\varphi_{n}\right) \geq B_{n}, n \in \mathrm{N},|F(\psi)| \leq\|\psi\|_{\infty} r(r I-U)^{-1}, \forall \psi \in X \\
\|F\| \leq \frac{r}{r-\|U\|}
\end{gathered}
$$

2) the following relations hold

$$
0 \leq B_{n} \leq U^{n}, \quad n \in \mathrm{N}
$$

3) the following inequalities hold

$$
B_{n} \leq r^{n+1}(r I-U)^{-1}, \quad n \in \mathrm{N}
$$

Then 2) $\Rightarrow$ 1) $\Rightarrow 3$ ).

Proof. 2) $\Rightarrow 1$ ). One applies theorem 2.1,2) implies 1), to $x_{j}=\varphi_{j}, j \in \mathrm{N}$. If

$$
\sum_{j \in J_{0}} \lambda_{j} \varphi_{j} \leq \psi=\sum_{n \in \mathrm{N}} \alpha_{n} \varphi_{n}, \lambda_{j} \in R_{+}
$$

then the hypothesis, Cauchy inequalities and the above relation yield:

$$
\begin{aligned}
& 0 \leq \lambda_{j} B_{j} \leq \lambda_{j} U^{j} \leq\left|\alpha_{j}\right| U^{j}, j \in \mathrm{N} \\
& \Rightarrow \sum_{j \in J_{0}} \lambda_{j} B_{j} \leq \sum_{j \in J_{0}}\left|\alpha_{j}\right| U^{j} \leq \sum_{n \in N} \frac{\|\psi\|_{\infty}}{r^{n}} U^{n} \\
& =\|\psi\|_{\infty}\left(I-\frac{U}{r}\right)^{-1}=r(r I-U)^{-1}\|\psi\|_{\infty}=: P(\psi)=P(-\psi)
\end{aligned}
$$


Hence, the implication of 2), Theorem 2.1 is accomplished and an application of the latter theorem leads to the existence of a linear positive operator $F$ applying $X$ into $Y$, with the properties stated at point 1$)$ :

$$
F\left(\varphi_{n}\right) \geq B_{n}, n \in \mathbb{N},|F(\varphi)| \leq r(r I-U)^{-1}\|\varphi\|_{\infty}, \varphi \in X
$$

Since the norm on $Y$ is solid, we infer that

$$
\|F(\varphi)\| \leq\left\|(I-U / r)^{-1}\right\| \cdot\|\varphi\|_{\infty}, \varphi \in X
$$

In particular, the following evaluation for the norm of $F$ holds

$$
\|F\| \leq\left\|\sum_{n=0}^{\infty} \frac{U^{n}}{r^{n}}\right\| \leq \sum_{n=0}^{\infty} \frac{\|U\|^{n}}{r^{n}}=\frac{r}{r-\|U\|}
$$

On the other hand, 1) $\Rightarrow 3$ ) is almost obvious, because of:

$$
B_{n}=F\left(\varphi_{n}\right) \leq\left\|\varphi_{n}\right\|_{\infty} r(r I-U)^{-1}=r^{n+1}(r I-U)^{-1}
$$

and $\varphi_{n} \in X_{+}$for all $n \in \mathrm{N}$. The conclusion follows.

Theorem 2.2.Let $X=L_{v}^{1}(M), v \geq 0$ and $\left(\varphi_{n}\right)_{n \in \mathrm{N}}$ a sequence of positive functions in $X$, such that $\int \varphi_{n} \mathrm{~d} v=1, \forall n \in \mathrm{N}$. Let $Y=L_{\mu}^{\infty}(\Omega), \mu \geq 0,\left(y_{n}\right)_{n \in \mathrm{N}}$ a sequence of positive functions in $Y$. Then $\sup _{n \in \mathrm{N}}\left(\left\|y_{n}\right\|\right)=b<\infty$ if and only if there is a linear positive operator $F \in L(X, Y)$ such that

$$
F\left(\varphi_{n}\right) \geq\left|y_{n}\right|, n \in \mathrm{N}, \quad|F(\psi)| \leq b \cdot\left(\int_{M}|\psi| \mathrm{d} v\right) \cdot \chi_{\Omega}, \forall \psi \in X
$$

Proof. For the "only if" part, let $J_{0} \subset \mathrm{N}$ be a finite subset, $\left\{\lambda_{j}\right\}_{j \in J_{0}} \subset R_{+}$be such that $\sum \lambda_{j} \varphi_{j} \leq \psi$ in $X$. Hypothesis on the functions $\varphi_{n}, n \in \mathrm{N}$ and integration in the relation $\sum_{j \in J_{0}} \lambda_{j} \varphi_{j} \leq \psi$ yield

$$
\begin{aligned}
& \sum_{j \in J_{0}} \lambda_{j}=\sum_{j \in J_{0}} \lambda_{j} \int_{M} \varphi_{j} \mathrm{~d} v \leq \int_{M} \psi \mathrm{d} v \\
& \Rightarrow \sum_{j \in J_{0}} \lambda_{j}\left|y_{j}\right| \leq\left\|\sum_{j \in J_{0}} \lambda_{j}\left|y_{j}\right|\right\| \cdot \chi_{\Omega} \leq\left(\int_{M} \psi \mathrm{d} v\right) \cdot b \cdot \chi_{\Omega} \\
& \leq\left(\int_{M}|\psi| \mathrm{d} v\right) \cdot b \cdot \chi_{\Omega}=: P(\psi)=P(-\psi), \psi \in X
\end{aligned}
$$

Application of theorem 2.1 leads to the existence of a linear positive operator $F \in L(X, Y)$ with the following properties

$$
F\left(\varphi_{n}\right) \geq\left|y_{n}\right|, n \in \mathrm{N}, \quad|F(\psi)| \leq b \cdot\left(\int_{M}|\psi| \mathrm{d} v\right) \cdot \chi_{\Omega}, \forall \psi \in X
$$

In particular, one has $\|F\| \leq b$. Next we prove the "if" part. Assume that $\left|y_{n}\right| \leq F\left(\varphi_{n}\right), n \in \mathrm{N}$ and $F$ has the qualities in the statement, then, because the norm on $Y$ is solid, we derive

$$
\left\|y_{n}\right\| \leq\left\|F\left(\varphi_{n}\right)\right\| \leq b\left(\int_{M} \varphi_{n} \mathrm{~d} v\right)=b, \quad n \in \mathrm{N}
$$

This concludes the proof. 


\section{On Markov Moment Problem}

We recall an earlier result on the abstract Markov moment problem, in order to apply it to the multidimensional classical moment problem.

Theorem 3.1. (Theorem 4 [10]). Let $X$ be a preordered linear space, $Y$ an order-complete vector lattice, $\left\{x_{j} ; j \in J\right\} \subset X,\left\{y_{j} ; j \in J\right\} \subset Y$ given finite or infinite families of elements, $F_{1}, F_{2} \in L(X, Y)$ two linear operators. The following statements are equivalent

1) there exists a linear operator $F \in L(X, Y)$ such that

$$
F\left(x_{j}\right)=y_{j} \forall j \in J, F_{1}(x) \leq F(x) \leq F_{2}(x) \forall x \in X_{+}
$$

2) for any finite subset $J_{0} \subset J$ and any $\left\{\lambda_{j} ; j \in J_{0}\right\} \subset R$, we have

$$
\sum_{j \in J_{0}} \lambda_{j} x_{j}=\psi_{2}-\psi_{1}, \psi_{l} \in X_{+}, l=1,2 \Rightarrow \sum_{j \in J_{0}} \lambda_{j} y_{j} \leq F_{2}\left(\psi_{2}\right)-F_{1}\left(\psi_{1}\right)
$$

The next result is quite similar to that of theorem 2.2.

Theorem 3.2. Let $X, Y,\left(\varphi_{n}\right)_{n},\left(y_{n}\right)_{n}$ be as in Theorem 2.2, and $0<b<\infty$; consider the following statements:

1) there exists a linear positive operator $F \in L(X, Y)$ such that

$$
F\left(\varphi_{n}\right)=y_{n}, n \in \mathrm{N},|F(\psi)| \leq b \cdot \int_{M}|\psi| \mathrm{d} v \cdot \chi_{\Omega}, \psi \in X,\|F\| \leq b
$$

2) for any finite subset $J_{0} \subset \mathrm{N}$ and any $\left\{\lambda_{j}\right\}_{j \in J_{0}} \subset R$, the following relation holds

$$
\sum_{j \in J_{0}}\left|\lambda_{j}\right| \cdot\left\|y_{j}\right\| \leq b\left|\sum_{j \in J_{0}} \lambda_{j}\right|
$$

Then 2) $\Rightarrow$ 1).

Proof. We apply Theorem 3.1,2) implies 1). If $\sum_{j \in J_{0}} \lambda_{j} \varphi_{j}=\psi_{2}-\psi_{1}$, where $\psi_{1}, \psi_{2} \in X_{+}$, then the following implications hold

$$
\begin{aligned}
& -\int_{M} \psi_{1} \mathrm{~d} v \leq \sum_{j \in J_{0}} \lambda_{j} \int_{M} \varphi_{j} \mathrm{~d} v=\sum_{j \in J_{0}} \lambda_{j} \leq \int_{M} \psi_{2} \mathrm{~d} v \\
& \Rightarrow\left|\sum_{j \in J_{0}} \lambda_{j}\right| \leq \int_{M} \psi_{2}+\int_{M} \psi_{1}=\int_{M} \psi_{2} \mathrm{~d} v-\left(-\int_{M} \psi_{1} \mathrm{~d} v\right)
\end{aligned}
$$

Now the hypothesis 2) yields

$$
\begin{aligned}
& \left|\sum_{j \in J_{0}} \lambda_{j} y_{j}\right| \leq\left.\left|\sum_{j \in J_{0}} \lambda_{j} y_{j}\right|\right|_{\infty} \cdot \chi_{\Omega} \leq\left(\sum_{j \in J_{0}}\left|\lambda_{j}\right| \cdot\left\|y_{j}\right\|\right) \cdot \chi_{\Omega} \\
& \leq b \cdot\left|\sum_{j \in J_{0}} \lambda_{j}\right| \chi_{\Omega} \leq b\left(\int_{M} \psi_{2} \mathrm{~d} v \cdot \chi_{\Omega}-\left(-\int_{M} \psi_{1} \mathrm{~d} v \cdot \chi_{\Omega}\right)\right) \\
& =F_{2}\left(\psi_{2}\right)-F_{1}\left(\psi_{1}\right), F_{2}(\psi):=b \int_{M} \psi \mathrm{d} v \cdot \chi_{\Omega}, F_{1}:=-F_{2}
\end{aligned}
$$

Application of theorem 3.1 leads to the existence of a linear operator $F \in L(X, Y)$ such that 


$$
\begin{aligned}
& F\left(\varphi_{n}\right)=y_{n}, n \in \mathrm{N}, \quad F_{1}(\psi)=-b \int_{M} \psi \mathrm{d} v \cdot \chi_{\Omega} \leq F(\psi) \leq F_{2}(\psi) \\
& =b \int_{M} \psi \mathrm{d} v \cdot \chi_{\Omega}, \psi \in X_{+} \Leftrightarrow|F(\psi)| \leq b \int_{M} \psi \mathrm{d} v \cdot \chi_{\Omega}, \psi \in X_{+} \\
& \Rightarrow|F(\varphi)| \leq\left|F\left(\varphi^{+}\right)\right|+\left|F\left(\varphi^{-}\right)\right| \leq b \cdot \int_{M}\left(\varphi^{+}+\varphi^{-}\right) \mathrm{d} v \chi_{\Omega} \\
& =b \int_{M}|\varphi| \mathrm{d} v \chi_{\Omega}, \varphi \in X
\end{aligned}
$$

This concludes the proof.

One goes on with an application of Theorem 3.1 to the operator valued real multidimensional moment problem. Let $X$ be the space of power series in $n$ complex variables, absolutely convergent in the polydisc $D=\prod_{k=1}^{n}\left\{\left|z_{k}\right| \leq r_{k}\right\}$, with real coefficients. The order relation on $X$ is defined by means of the coefficients, similar to the case of Proposition 2.1. Let $H$ be a complex Hilbert space, $A_{k}, k=1, \cdots, n$ linear positive self-adjoint commuting operators on $H$, such that $\left\|A_{k}\right\| \leq r_{k}, k=1, \cdots, n$. We denote:

$$
Y_{1}=\left\{U \in \mathrm{A}(H) ; A_{k} U=U A_{k}, k=1, \cdots, n\right\}, Y=\left\{V \in Y_{1} ; V U=U V \forall U \in Y\right\}
$$

Here $\mathrm{A}(H)$ is the real vector space of all selfadjoint operators acting on $H$. Then $Y$ is an order complete Banach lattice [14] and clearly is a commutative Banach algebra of selfadjoint operators. Denote

$$
\varphi_{j}\left(z_{1}, \cdots, z_{n}\right)=z_{1}^{j_{1}} \cdots z_{n}^{j_{n}}, j=\left(j_{1}, \cdots, j_{n}\right) \in \mathrm{N}^{n}
$$

Theorem 3.3. Let $\left(B_{j}\right)_{j \in \mathbb{N}^{n}}$ be a sequence in $Y, b>0$ a real number. The following statements are equivalent

1) there exists a linear operator $F \in L(X, Y)$ such that

$$
F\left(\varphi_{k}\right)=B_{k}, k \in \mathrm{N}^{n}, 0 \leq F(\psi) \leq b \psi\left(A_{1}, \cdots, A_{n}\right), \forall \psi \in X_{+}
$$

2) the following relations hold

$$
0 \leq B_{k} \leq b A_{1}^{k_{1}} \cdots A_{n}^{k_{n}}, \forall k=\left(k_{1}, \cdots, k_{n}\right) \in \mathbb{N}^{n}
$$

Proof. The implication 1) $\Rightarrow 2$ ) is almost obvious, since $\varphi_{k} \in X_{+}, k \in \mathbb{N}^{n}$ and the hypothesis 1) yields

$$
B_{k}=F\left(\varphi_{k}\right) \in\left[0, b \varphi_{k}\left(A_{1}, \cdots, A_{n}\right)\right]=\left[0, b A_{1}^{k_{1}} \cdots A_{n}^{k_{n}}\right]
$$

For the converse, we apply Theorem 3.1,2) implies 1). Assume that

$$
\sum_{j \in J_{0}} \lambda_{j} \varphi_{j}=\psi_{2}-\psi_{1}=\sum_{k \in \mathbb{N}^{n}} \alpha_{k} \varphi_{k}-\sum_{k \in \mathbb{N}^{n}} \beta_{k} \varphi_{k}, \alpha_{k}, \beta_{k} \in R_{+}, k \in \mathrm{N}^{n}
$$

From these relations we derive

$$
\lambda_{j} \leq \alpha_{j}, j \in J_{0}
$$

which further yields

$$
\sum_{j \in J_{0}} \lambda_{j} B_{j} \leq \sum_{j \in J_{0}} \alpha_{j} B_{j} \leq \sum_{k \in \mathbb{N}^{n}} \alpha_{k} B_{k} \leq b \sum_{k \in \mathbb{N}^{n}} \alpha_{k} A_{1}^{k_{1}} \cdots A_{n}^{k_{n}}=F_{2}\left(\psi_{2}\right)-F_{1}\left(\psi_{1}\right)
$$

Thus the implication from 2), Theorem 3.1 is verified, where 


$$
F_{2}\left(\sum_{k \in \mathbb{N}^{n}} \alpha_{k} \varphi_{k}\right):=b \sum_{k \in \mathbb{N}^{n}} \alpha_{k} A_{1}^{k_{1}} \cdots A_{n}^{k_{n}}, F_{1}:=0
$$

Application of the latter theorem leads to the existence of a linear operator $F \in L(X, Y)$ satisfying the moment conditions $F\left(\varphi_{k}\right)=B_{k}, k \in \mathrm{N}^{n}$, such that on the positive cone of $X$, the following relations hold

$$
0=F_{1}(\psi) \leq F(\psi) \leq F_{2}(\psi)=b \psi\left(A_{1}, \cdots, A_{n}\right), \quad \psi \in X_{+}
$$

This concludes the proof.

We go on with applications related to a convexity and extension of linear operators result, having a nice geometric meaning. If $V$ is a convex neighborhood of the origin in a locally convex space, we denote by $p_{V}$ the gauge attached to $V$.

Theorem 3.4. (see [9]). Let $X$ be a locally convex space, $Y$ an order complete vector lattice with strong order unit $u_{0}$ and $S \subset X$ a vector subspace. Let $A \subset X$ be a convex subset with the following qualities:

1) there exists a neighborhood $V$ of the origin such that

$$
(S+V) \cap A=\Phi
$$

(that is, by definition, $A$ and $S$ are distanced);

2) $A$ is bounded.

Then for any equicontinuous family of linear operators $\left\{f_{j}\right\}_{j \in J} \subset L(S, Y)$ and for any $\tilde{y} \in Y_{+} \backslash\{0\}$, there exists an equicontinuous family $\left\{F_{j}\right\}_{j \in J} \subset L(X, Y)$ such that

$$
F_{j}(s)=f_{j}(s), s \in S \text { and } F_{j}(\psi) \geq \tilde{y}, \psi \in A, j \in J
$$

Moreover, if $V$ is a neighborhood of the origin such that

$$
f_{j}(V \cap S) \subset\left[-u_{0}, u_{0}\right],(S+V) \cap A=\Phi
$$

and if $\alpha>0$ is such that $p_{V}(a) \leq \alpha \forall a \in A$, while $\alpha_{1}>0$ is large enough such that $\tilde{y} \leq \alpha_{1} u_{0}$, then the following relations hold

$$
F_{j}(x) \leq\left(1+\alpha+\alpha_{1}\right) p_{V}(x) \cdot u_{0}, \quad x \in X, j \in J
$$

Recall that the definition and terminology of distanced (convex) subsets written above is motivated by the fact that in the particular case when $X$ is a normed vector space, the neighborhood $V$ appearing in relation (3.18) can be chosen as a ball centered at the origin. Then (3.18) is equivalent to the relation $d(S, A) \geq r>0$, where $d(S, A)$ is the distance between the two subsets $S$ and $A$. with respect to the metric defined by the norm on $X$, and $r$ is the radius of that ball. In this particular case, $V$ can be chosen as $V=B(0, r)$, for some $r>0$ sufficiently small. If $V$ is a convex circled neighborhood of zero in a locally convex space, one can define $d_{V}(x, y):=p_{V}(x-y)$, where $p_{V}$ is the gauge attached to $V$. Then $d_{V}$ has all the properties of a distance defined by means of a norm, except one of them. Precisely, $d_{V}\left(x_{1}, x_{2}\right)=0$ does not imply $x_{1}=x_{2}$, since $p_{V}$ is just a seminorm, not a norm. On the other hand, it is the case when $X$ is a normed vector space to which Theorem 3.4 will be applied in the sequel. Namely, in the next 
theorem $X$ will be the space $C\left(\left[0, b_{1}\right] \times \cdots \times\left[0, b_{n}\right]\right), \quad Y=Y\left(A_{1}, \cdots, A_{n}\right)$ is the space defined by (3.8) ( $A_{1}, \cdots, A_{n}$ are commuting positive selfadjoint operators), under the additional assumption $\sigma\left(A_{k}\right)=\left[0, b_{k}\right], k=1, \cdots, n$,

$$
\begin{aligned}
& \varphi_{j} \in X, \varphi_{j}\left(t_{1}, \cdots, t_{n}\right)=t_{1}^{j_{1}} \cdots t_{n}^{j_{n}},\left(t_{1}, \cdots, t_{n}\right) \in\left[0, b_{1}\right] \times \cdots \times\left[0, b_{n}\right], \\
& j=\left(j_{1}, \cdots, j_{n}\right) \in \mathrm{N}^{n},|j|:=\sum_{k=1}^{n} j_{k} \geq 1
\end{aligned}
$$

Theorem 3.5. Let $\left(\psi_{j}\right)_{j \in \mathrm{N}^{n}}$ be a sequence in $X$ such that $\psi_{j}(0, \cdots, 0)=1$ $\psi_{(0, \cdots, 0)} \equiv 1,\left\|\psi_{j}\right\|_{\infty} \leq 1$ for all $j \in \mathrm{N}^{n}$, and let $B \in Y, B \geq I$. Then there exists a linear bounded positive operator $F \in B(X, Y)$, which is multiplicative on the subspace of continuous functions vanishing at the origin, such that

$$
\begin{aligned}
& F\left(\varphi_{j}\right)=A_{1}^{j_{1}} \cdots A_{n}^{j_{n}}, j \in \mathrm{N}^{n},|j| \geq 1, \\
& F\left(\psi_{j}\right) \geq B, \forall j \in \mathrm{N}^{n},|F(\varphi)| \leq(2+\|B\|) \cdot\|\varphi\| \cdot I, \forall \varphi \in X
\end{aligned}
$$

Proof. Denote $A=\operatorname{conv}\left\{\psi_{j} ; j \in \mathrm{N}^{n}\right\}, S=\operatorname{Span}\left(\left\{\varphi_{j} ;|j| \geq 1\right\}\right)$. Then we get

$$
\begin{aligned}
& \|s-a\|_{\infty} \geq|s(0)-a(0)|=1, \forall s \in S, \forall a \in A \\
& \Rightarrow(S+B(0,1)) \cap A=\Phi, V:=B(0,1),\|\psi\|_{\infty} \leq 1=: \alpha, \forall \psi \in A
\end{aligned}
$$

Thus, the unit ball $B(0,1)$ of the space $X$ stands for $V$ of the preceding theorem 3.4, \|\| stands for $p_{V}$, and $A$ is the convex hull of the collection of functions $\psi_{j}, j \in \mathrm{N}^{n}$. Define

$$
f: S \rightarrow Y, f(s)=f\left(\sum_{j \in J_{0}} a_{j} \varphi_{j}\right)=\sum_{j \in J_{0}} a_{j} A_{1}^{j_{1}} \cdots A_{n}^{j_{n}}
$$

where $J_{0} \subset\left\{j \in N^{n} ;|j| \geq 1\right\} \subset N^{n}$ is a finite subset. If $s \in S \cap B(0,1)$, then

$$
\begin{aligned}
& -1 \leq s=\sum_{j \in J_{0}} a_{j} t_{1}^{j_{1}} \cdots t_{n}^{j_{n}} \leq 1 \quad \forall\left(t_{1}, \cdots, t_{n}\right) \in\left[0, b_{1}\right] \times \cdots \times\left[0, b_{n}\right] \\
& \Rightarrow-I \leq f(s)=\sum_{j \in J_{0}} a_{j} A_{1}^{j_{1}} \cdots A_{n}^{j_{n}} \leq I
\end{aligned}
$$

because of the positivity of the spectral measures associated to the $n$-tuple $\left(A_{1}, \cdots, A_{n}\right)$. On the other hand $B \leq\|B\| \cdot I$, so that all conditions of theorem 3.4 are verified for

$$
\alpha_{1}=\|B\|, \alpha=1, u_{0}=I
$$

Application of theorem 3.4 leads to the existence of a linear extension $F$ of $f$, such that

$$
\begin{aligned}
& F(\varphi) \leq(2+\|B\|)\|\varphi\|_{\infty} I, \\
& \forall \varphi \in X \Rightarrow|F(\varphi)| \leq(2+\|B\|) \cdot\|\varphi\|_{\infty} I \Rightarrow\|F\| \leq 2+\|B\|, \\
& F\left(\psi_{j}\right) \geq B:=\tilde{y}, \forall j \in \mathrm{N}^{n} \Rightarrow F(1) \geq B
\end{aligned}
$$

In particular, $F$ is continuous. Now we prove that $F$ is also positive. Let $p$ be a polynomial

$$
p\left(t_{1}, \cdots, t_{n}\right)=\sum_{j \in J_{1}} a_{j} t_{1}^{j_{1}} \cdots t_{n}^{j_{n}} \geq 0 \quad \forall\left(t_{1}, \cdots, t_{n}\right) \in\left[0, b_{1}\right] \times \cdots \times\left[0, b_{n}\right]
$$


where $J_{1} \subset \mathrm{N}^{n}$ is a finite subset. Then using the positivity of the spectral measures attached to $n$-tuple of operators $\left(A_{1}, \cdots, A_{n}\right)$, as well as the relations

$$
F(1)=F\left(\psi_{(0, \cdots, 0)}\right) \geq B \geq I, a_{(0, \cdots, 0)}=p(0, \cdots, 0) \geq 0
$$

we derive the following implications

$$
\begin{aligned}
& s:=\sum_{j \in J_{1}, j \mid j \geq 1} a_{j} t_{1}^{j_{1}} \cdots t_{n}^{j_{n}} \geq-a_{(0, \cdots, 0)} \Rightarrow F(s)=\sum_{j \in J_{1},|j| \geq 1} a_{j} A_{1}^{j_{1}} \cdots A_{n}^{j_{n}} \geq-a_{(0, \cdots, 0)} \cdot I \\
& \Rightarrow F(p)=a_{(0, \cdots, 0)} F(1)+F(s) \geq a_{(0, \cdots, 0)}(F(1)-I) \geq a_{(0, \cdots, 0)}(B-I) \geq 0
\end{aligned}
$$

Application of Weierstrass approximation theorem and the continuity of $F$ lead to the positivity of $F$ on $X$.

Hypothesis on the fact that $A_{1}, \cdots, A_{n}$ are permutable and a straightforward computation shows that

$$
f\left(p_{1} p_{2}\right)=f\left(p_{1}\right) f\left(p_{2}\right)
$$

for all polynomials of $n$ variables, vanishing at the origin. Since $F$ is a continuous linear extension of $f$ and the product operation on the Banach algebra $Y$ is continuous, we infer that $F$ is multiplicative on the subspace of continuous functions vanishing at the origin (use Bernstein approximating polynomials of $n$ variables: if a continuous function vanishes at the origin, then all the corresponding Bernstein polynomials do the same). This concludes the proof.

\section{Conclusions}

In the first part of this work, new applications of Mazur-Orlicz theorem have been proved (Section 2). In Section 3, Markov type moment problem results are studied. Comparing theorems 2.2 and 3.2, we see that the proofs of the two type-problems mentioned above are different, even in cases of similar statements. The last result of the paper is an application of an earlier theorem. The new element with respect to previous submissions is that here the solution is defined on a space of continuous functions of several real variables, vanishing at the origin (see Theorem 3.5). Our solutions are operator or function valued. Further applications could be deduced, depending on the knowledge and imagination of the authors.

\section{Acknowledgements}

The authors would like to thank the anonymous referee for reading carefully the manuscript and for his meaningful suggestions, leading to the improvement of the presentation of this paper.

\section{References}

[1] Kőnig, H. (1982) On Some Basic Theorems in Convex Analysis. In: Korte, B., Ed., Modern Applied Mathematics-Optimization and Operations Research, North Holland, 107-144.

[2] Neumann, M.M. (1991) Generalized Convexity and the Mazur-Orlicz Theorem. Proceedings of the Orlicz Memorial Conference, University of Mississippi, 21-23 
March 1991, 1-15.

[3] Olteanu, O. and Mihăilă, J.M. (2017) Operator-Valued Mazur-Orlicz and Moment Problems in Spaces of Analytic Function. UPB Scientific Bulletin, Series A, 79, 175-184.

[4] Olteanu, O. (2015) Markov Moment Problem and Related Approximation. Mathematical Reports, 17, 107-117.

[5] Olteanu, O. and Olteanu, A. (2014) Applications of Extension Theorems of Linear Operators to Mazur-Orlicz and Moment Problems. UPB Scientific Bulletin, Series $A, 76,31-42$.

[6] Olteanu, O. (2013) New Results on Markov Moment Problem. International Journal of Analysis, 2013, Article ID: 901318.

[7] Lemnete-Ninulescu, L. and Olteanu, O. (2017) Applications of Classical and Functional Analysis. Lambert Academic Publishing, Saarbrücken.

[8] Olteanu, O. (1978) Convexité et prolongement d'opérateurs linéaires. [Convexity and Extension of Linear Operators.] Comptes Rendus de I Académie des Sciences Paris, Série A, 286, 511-514.

[9] Olteanu, O. (1983) Théorèmes de prolongement d'opérateurs linéaires. [Extension Theorems for Linear Operators.] Revue Roumaine de Mathématique Pures et Appliquées, 28, 953-983.

[10] Olteanu, O. (1991) Application de théorèmes de prolongement d'opérateurs linéaires au problème des moments et à une généralisation d'un théorème de $\mathrm{Ma}$ zur-Orlicz. [Applications of Extension Theorems for Linear Operators to the Moment Problem and to a Generalization of a Mazur-Orlicz Theorem.] Comptes Rendus de 1 Académie des Sciences Paris, Série I, 313, 739-742.

[11] Berg, C. and Duran, A.J. (2008) A Fixed Point for a Transformation of Hausdorff Moment Sequences and Iteration of a Rational Function. Mathematica Scandinavica, 103, 11-39. https://doi.org/10.7146/math.scand.a-15066

[12] Fuglede, B. (1983) The Multidimensional Moment Problem. Expositiones Mathematicae, 1, 47-65.

[13] Stoyanov, J. and Lin, G.D. (2013) Hardy's Condition in the Moment Problem for Probability Distributions. Theory of Probability and Its Applications, 57, 699-708.

[14] Cristescu, R. (1976) Ordered Vector Spaces and Linear Operators, Academiei, Bucharest. Romania and Abacus Press, Tunbridge Wells.

[15] Niculescu, C. and Popa, N. (1981) Elements of Theory of Banach Spaces. Academiei, Bucharest. (In Romanian)

[16] Rudin, W. (1999) Real and Complex Analysis. 3rd Edition, Theta, Bucharest. (In Romanian) 
Submit or recommend next manuscript to OALib Journal and we will provide best service for you:

- Publication frequency: Monthly

- 9 subject areas of science, technology and medicine

- Fair and rigorous peer-review system

- Fast publication process

- Article promotion in various social networking sites (LinkedIn, Facebook, Twitter, etc.)

- Maximum dissemination of your research work

Submit Your Paper Online: Click Here to Submit

Or Contact service@oalib.com 